Estudios Románicos, Volumen 30, 2021, pp. 39-58

ISSN: 0210-4911

eISSN: $1989-614 \mathrm{X}$

DOI: https://doi.org/10.6018/ER.471241

\title{
MÁS TONTOS QUE EL NOVIO DE LA CHELO. LA INTENSIFICACIÓN DE LA ESTULTICIA EN FOROS Y CHATS POR MEDIO DE COMPARACIONES CREATIVAS: UNA APROXIMACIÓN DESDE LA GRAMÁTICA DE CONSTRUCCIONES
}

(Más tontos que el novio de la Chelo. The intensification of stupidity in forums and chats by means of creative comparisons: a Construction Grammar approach)

\author{
Pedro Ivorra Ordines* \\ Universitat Pompeu Fabra \\ Carmen Mellado Blanco** \\ Universidade de Santiago de Compostela
}

\begin{abstract}
Adopting a corpus-based approach, the main aim of this study is to inductively describe the intensifying comparative construction [ser más tonto que $\mathrm{X}$ ]: 'be foolish in its highest degree'. Therefore, it will be applied the Construction Grammar approach. In this sense, lexical fillers of the slot $\mathrm{X}$ will be quantitatively and qualitatively analysed in order to determine the entrenchment and productivity of the construction, as well as to find out potential recurrent traits of its creativity. This study aims to show the fine line between lexicalised and creative units, the latter being the most predominant.
\end{abstract}

Keywords: Intensification; Productivity; Entrenchment; Intensifying comparative construction.

Resumen: El objetivo principal de este trabajo consiste en describir por medio de corpus, con una metodología inductiva, la construcción comparativa intensificadora

* Dirección para correspondencia: Pedro Ivorra Ordines. Departament de Traducció i Ciències del Llenguatge. Facultat de Traducció i Ciències del Llenguatge. Universitat Pompeu Fabra. Roc de Boronat 138. 08018 Barcelona (pedro.ivorra@upf.edu).

${ }^{* *}$ Dirección para correspondencia: Carmen Mellado Blanco. Departamento de Filología Inglesa y Alemana. Facultad de Filología. Universidad de Santiago de Compostela. Avda. de Castelao s/n. 15704 Santiago de Compostela (c.mellado@usc.es). 
[ser más tonto que $\mathrm{X}$ ]: 'ser tonto en grado sumo', para lo que se tomará como marco teórico la Gramática de Construcciones. En este sentido se realizará un estudio cuantitativo y cualitativo de las actualizaciones léxicas del slot $\mathrm{X}$ con el fin de determinar la fijación cognitiva y la productividad de la construcción, así como descubrir posibles rasgos recurrentes en su creatividad. Con esta investigación intentamos demostrar cuán fina es la línea que separa las unidades lexicalizadas de las creativas, con un predominio claro de estas últimas.

Palabras clave: Intensificación; Productividad; Fijación cognitiva; Construcción comparativa intensificadora.

\section{Introducción: las construcciones comparativas intensificadoras ${ }^{1}$}

De acuerdo con Albelda Marco (2007: 114), la intensificación es una categoría pragmática compleja que tiene carácter escalar, es decir, se trata de una forma de gradación de una cualidad o cantidad a la que el hablante alude en un acto de habla. Desde un punto de vista comunicativo tiene una función claramente evaluativa, a través de la cual el hablante emite un juicio de valor o una apreciación. Socialmente, la intensificación está destinada a aumentar la eficiencia comunicativa, es decir, a hacer más creíble lo dicho, a imponer el yo y a buscar el acuerdo del oyente. El objeto de investigación de este trabajo, las comparaciones intensificadoras, comparte las características descritas en este enfoque tridimensional de la intensificación. En efecto, estas comparaciones tienen un valor escalar, ya que sirven, en primer lugar, para establecer una gradación con respecto a la cualidad denotada. Desempeñan, además, una función evaluativa para el hablante y sirven para aumentar la eficacia comunicativa de cara al oyente. Por todo ello, las comparaciones intensificadoras, como su nombre bien indica, sirven para intensificar. Con ello se distancian de las comparaciones gramaticales, del tipo Pedro es más alto que Marta, destinadas a designar una gradación de manera objetiva, y no a evaluar subjetivamente sobre la magnitud o intensidad de una cualidad o un estado. A diferencia de estas, las comparaciones del tipo Tu hermano es más tonto que Abundio (significado: 'tu hermano es tonto en grado sumo'), cumplen con el objetivo de intensificar el contenido proposicional y presentan modalidad epistémica. Por otra parte, es interesante señalar que los cuantificadores relativos, como más o menos (Albelda Marco 2007: 23), cuya función es la de establecer una relación entre dos objetos con respecto al grado de una cualidad, dejan de ejercer tal función en la comparación intensificadora, ya que el segundo término de la comparación carece de significado referencial (cfr. Mellado Blanco 2015 y 2019).

El binomio 'intensificación'/'comparación' en español ha sido investigado hasta la fecha básicamente desde un punto de vista fraseológico, es decir, en su vertiente de

1 Este trabajo ha surgido en el marco del proyecto de investigación PID2019-108783RB-I00: Gramática de Construcciones y Fraseología. Las construcciones fraseológicas del alemán y el español en contraste a través de los corpus. El proyecto, financiado por el Ministerio de Ciencia e Innovación, se desarrolla en el seno del grupo de investigación FRASESPAL bajo la dirección de Carmen Mellado Blanco. 
Más tontos que el novio de la Chelo. La intensificación de la estulticia en foros y chats por medio de comparaciones creativas: una aproximación desde la gramática de construcciones

comparaciones estereotipadas ${ }^{2}$, como recurso lingüístico "prefabricado" de la lengua del que los hablantes se sirven para intensificar una cualidad, un estado o una acción que vienen expresados por un adjetivo o verbo en función del tertium comparationis. De esta manera, en la comparación fraseológica más sordo que una tapia, el tertium comparationis es el adjetivo sordo, y en beber como un cosaco es el verbo beber el que ejerce como tertium comparationis (cfr. Mellado Blanco 2012a). En estas comparaciones, el comparatum o segundo término de la comparación representa el prototipo en grado sumo de lo denotado por el tertium comparationis y está así convencionalizado en la comunidad lingüística.

Dejando a un lado la investigación fraseológica, son pocos los estudios que abordan el binomio 'intensificación'/‘comparación' desde un prisma más amplio, es decir, que no vinculen necesariamente la intensificación con el estereotipo convencionalizado y lexicalizado. Como veremos en nuestro estudio de corpus de [ser más tonto que X]: 'ser tonto en grado sumo', las comparaciones intensificadoras empleadas en el discurso son, en su mayoría, formas creativas no registradas lexicográficamente, que, no obstante, cumplen con los requisitos necesarios para considerarse como instancias de una construcción comparativa intensificadora desde una perspectiva construccionista. En este sentido, la Gramática de Construcciones se presenta como un marco de estudio idóneo para tratar el tema de las comparaciones intensificadoras en toda su amplitud y complejidad creativa.

En cuanto a la elección de género textual, nos hemos decantado por los chats y foros por ser buenos representantes de la oralidad conceptual (Koch y Oesterreicher 1985), esto es, del discurso inmediato, informal, cercano y espontáneo, independientemente de que se desarrolle por medio de un canal escrito. Las realizaciones discursivas de las construcciones comparativas intensificadoras se caracterizan por su espontaneidad y expresividad y son características de la oralidad. Dada la escasez de corpus orales que permitan un estudio integral de este tipo de construcciones, los chats y foros se presentan como un género discursivo digital ideal para analizar este tipo de unidades (cfr. Kleinberger Günther 2006; Stein 2010; Mellado Blanco 2012b)3․ Así pues, un breve repaso por las fuentes de las instancias de la construcción pone de manifiesto que un $71,98 \%$ de las ocurrencias proviene de foros y chats, tratándose de las fuentes con una mayor incidencia en el corpus, seguido de, con menor presencia, periódicos y revistas digitales $(11,91 \%)$ y otras fuentes como recopilaciones de comparaciones fraseológicas,

2 Para una bibliografía detallada de la comparación fraseológica (vid. Penadés Martínez 2012: 130-134; Ivorra Ordines2021). Las comparaciones estereotipadas son consideradas por Albelda Marco (2007: 58) como un procedimiento léxico-fraseológico de intensificación proposicional, en concreto como locuciones adjetivales, al tiempo que son estudiadas también como un tipo de esquema sintáctico intensificador (2007: 71-72). En este sentido, la autora habla de relación entre dos miembros por la presencia del cuantificador más, menos o como (Albelda Marcos 2007: 71), cuando en realidad las comparaciones estereotipadas no son relativas, sino elativas por definición, puesto que el comparatum carece de significado referencial (vid. supra).

3 Los paralelismos entre chats y conversaciones orales son puestos de manifiesto en la investigación de Beißwenger (2003). En relación con los foros, si bien la producción y recepción de sus mensajes no discurre de manera sincrónica al cien por cien, a diferencia de los chats, también son una importante fuente de datos para el estudio de la oralidad conceptual. 
pequeños fragmentos de obras literarias, la enciclopedia electrónica Wikipedia o descripciones de archivos audio (16,11\%).

\section{La construcción comparativa intensificadora [más tonto que $\mathrm{X}$ ]}

\subsection{Caracterización de la construcción en el marco de la Gramática de Construcciones}

La construcción comparativa intensificadora es un tipo de construcción semiesquemática, es decir, presenta una saturación léxica solo parcial. Así, en nuestra construcción [ser más tonto que X]: 'ser muy tonto', los constituyentes fijos son más, tonto, que, mientras que el comparatum es un slot o casilla vacía que se actualiza en el discurso (p. ej. Pichote, un zapato, una mula, etc.). De acuerdo con la Gramática de Construcciones, las construcciones parcialmente saturadas desde un punto de vista léxico se encuentran a medio camino entre las construcciones sustantivas (cfr. Fillmore, Kay y O’Connor 1988: 505), es decir, constituidas por componentes fijos (locuciones, morfemas, monolexemas) y las construcciones formales, de estructura argumental. Como el resto de construcciones, las construcciones comparativas intensificadoras son emparejamientos de forma y significado en las que la propia estructura es portadora de un significado concreto (cfr. Goldberg 1995 y 2019). De esta manera, la construcción [más tonto que $\mathrm{X}$ ] está ligado, como construcción, al significado intensificador 'tonto en grado máximo', y ello independientemente de la actualización léxica concreta de $\mathrm{X}$ en el discurso. Aplicando a nuestra construcción el principio de coerción (cfr. Michaelis 2003), podríamos decir que el significado intensificador de la construcción "impregna" toda la estructura por encima del significado particular de sus componentes, lo cual afecta particularmente a X, cuyo significado extraconstruccional se diluye a favor del significado global intensificador. Por este motivo, la actualización léxica de $\mathrm{X}$ se ve privada de una interpretación literal y abarca un gran espectro de posibilidades creativas, como el que da título a nuestro trabajo más tontos que el novio de la Chelo.

(1) ¿No puedes opinar por ti mismo? Si a ti te ha gustado la película, ¿cómo puede dejarte de gustar por lo que opinen unos señores/as, a los que no conoces de nada y que posiblemente sean más tontos que el novio de la Chelo? Y vamos a ver: los señores/ as antiamericanos, ¿por qué van a ver películas americanas? [SkE 11341184194]

En este contexto, como apunta Cifuentes Honrubia (2019: 55-56), entrarían en funcionamiento las implicaciones pragmáticas del principio escalar para denotar lo más tonto posible, por lo que el novio de la Chelo, por el simple hecho de funcionar como comparatum en el constructo de la construcción comparativa intensificadora [más tonto que $\mathrm{X}$ ], pasaría automáticamente a ser arreferencial y a ocupar el lugar más alto de una escala imaginaria de acciones y personas tenidas por tontas (en el amplio sentido de la palabra) en el imaginario colectivo de los hablantes del español. Se trata de un conocimiento cultural y pragmático que hace posible que podamos entender dicha comparación, aunque sea la primera vez que la escuchemos. Desde esta perspectiva, 
Más tontos que el novio de la Chelo. La intensificación de la estulticia en foros y chats por medio de comparaciones creativas: una aproximación desde la gramática de construcciones

el comparatum entraña subjetivación al producirse una pragmatización creciente del significado que adquiere valores adicionales, teniendo en cuenta que es posible considerar el novio de la Chelo como el equivalente pragmático de persona representante o prototípica de la estulticia (cfr. Fauconnier 1975).

Como ya hemos apuntado más arriba, la comparación intensificadora, si bien coincide en la forma con la gramatical, se diferencia de esta en que no posee un significado composicional, como se observa en (3). Por el contrario, el ejemplo del corpus de comparación gramatical (2) presenta un significado literal en el que se comparan, efectivamente, dos elementos (un jabalí y tú) con respecto a la magnitud de la estulticia:

(2) Pero a ti no te la han colado, enhorabuena. Al jabalí sí, porque es más tonto que tú, miró google maps y pensó que eso era monte. [SkE 15063820242]

(3) Era más tonto que la hostia ese tío. [SkE 592194121]

Las instancias (2) y (3) están licenciadas por construcciones distintas, aunque coincidan en la forma general [A es más X que B]. No obstante, no siempre está claro si nos encontramos ante la actualización de la construcción gramatical o de la intensificadora, ya que las construcciones representan un fenómeno holístico dentro de un continuum léxico-gramatical. Tal ambigüedad se vislumbra en especial en los casos en los que el comparatum es un nombre propio, como en (4). En este ejemplo no podemos saber si hay que interpretar el comparatum (George McFly) literalmente, como un referente real o bien como prototipo de la estulticia (caso de que el ejemplo estuviera licenciado por la construcción intensificadora). En el último caso, la intencionalidad del hablante sería la de intensificar la cualidad de tonto en relación con el comparandum Forrest Gump, creando ad hoc un prototipo (George McFly) para ese determinado contexto.

(4) En cuanto a Robert Zemeckis, conoció a un primo de Marty llamado Forest Gump que era incluso más tonto que George McFly, y decidió rodar un documental sobre su vida. [SkE 15829183056]

De lo anterior se desprende que en la construcción [ser más tonto que X]: 'ser muy tonto', el slot $\mathrm{X}$ se concibe como el representante prototípico de la estulticia ${ }^{4}$. La actualización del slot puede tener lugar mediante comparata lexicalizados en esta función: Pichote, Abundio, el que asó la manteca, mandado a hacer de encargo (vid. 3 y 5), en ocasiones con alguna modificación o cruce (vid. 6), o bien mediante términos creados por el hablante (vid. 7).

4 Se observa algún caso en el corpus de comparación elidida, sin comparatum, o comparación suspendida con omisión del segundo término de la comparación (cfr. Albelda Marco 2007: 78). La elisión también se conoce bajo el nombre "aposiopesis" u omisión de una parte del enunciado por considerarse como obvia (cfr. Beinhauer 1985: 307). En el nivel prosódico se observa una curva de entonación ascendente después del truncamiento, que se mantiene en suspensión durante un breve intervalo (cfr. Mellado Blanco 2012a: 26). Desde un punto de vista construccionista, este tipo de elisiones corroboran el alto grado de fijación cognitiva de la construcción, cuyo truncamiento no es obstáculo para su correcta descodificación. Ejemplo del corpus: "Lo de la mantis religiosa es que la hembra es más mala que el sebo de gato y el macho es más tonto que...”. [SkE 5612846326] 
(5) El padre era un mal bicho, pero tenía un aquel. El hijo era más tonto que Abundio y no tenía nada de nada. [SkE 608210122]

(6) Somos más tontos que Abundio o Pichote. Como decía un oficial del ejército alemán. ¿Cómo quiere usted las órdenes, fritas o asadas? [SkE 15677579297]

(7) Sí, ya lo sé: soy más tonto que un boniato mohoso, pero nunca nunca nunca comprenderé cómo es posible que personas "de a pie" [...]. [SkE 17225733217]

A su vez, las actualizaciones creativas pueden ser el resultado de una extensión analógica a partir de comparaciones estereotipadas (p.ej. ser más tonto que hecho adrede, a partir de ser más tonto que mandado a hacer de encargo) o por el contrario pueden no mostrar relación aparente con estas (ejemplo 7). En el caso de las actualizaciones lexicalizadas del slot X, la estructura de X no es homogénea: nombres propios (Abundio, Pichote), sintagmas nominales con nombres comunes (la leche), oraciones de relativo (el que asó la manteca), oraciones comparativas irreales (mandado a hacer de encargo ${ }^{5}$ ).

Los comparata que no aparecen registrados en ningún diccionario no son siempre creaciones $a d h o c$, sino que más bien evidencian la inevitable parcialidad de los diccionarios. En este sentido, parafraseando a Manuel Seco (vid. Rodríguez 2000), constatamos la imposibilidad de plasmar en un diccionario toda la realidad de una lengua. Con ello nos referimos a las actualizaciones del slot X que pueden interpretarse en vías de lexicalización por su alta frecuencia token, como sucede con un obrero de derechas (54 ocurrencias en esTenTen18), no registrado lexicográficamente, pero con un alto grado de fijación cognitiva (entrenchment) y convencionalización en virtud de su frecuencia. Las variaciones que presenta esta microconstrucción emergente (vid. 8-9) son, asimismo, una prueba de su fijación cognitiva.

(8) Ya lo decía mi abuelo: eres más tonto que un currito del PP... [SkE 19325795512]

(9) ¿Hay algo más tonto que un currito votando a un partido de derecha? [SkE 560341261]

Por otra parte, las variaciones encontradas también en algunas de las locuciones lexicalizadas confirman la idea de que estas en realidad no son tan fijas en el plano formal, sino más bien en el cognitivo (cfr. Mellado Blanco 2020a).

\subsection{Corpus y productividad de la construcción}

Nuestro estudio se basa en las ocurrencias halladas en el corpus esTenTen $18^{6}$ de Sketch Engine a partir de la búsqueda de la secuencia <más tonto que>. En la búsqueda hemos considerado tonto como lema y por ello se han incluido todas las formas posibles (tonto, tonta, tontos, tontas), siendo la más frecuente tonto, con 1179 resultados. La búsqueda de

$5 \quad$ Este caso puede interpretarse como elisión de si hubiera sido mandado a hacer de encargo.

6 Se trata de un macrocorpus del español que contiene 20306642991 tokens. Gran parte de los textos recogidos corresponden a un registro informal, por lo que se prestan especialmente para estudiar las construcciones intensificadoras y la creatividad de estas. 
Más tontos que el novio de la Chelo. La intensificación de la estulticia en foros y chats por medio de comparaciones creativas: una aproximación desde la gramática de construcciones

la citada secuencia con el lema tonto arrojó un resultado final de 2387 ocurrencias, de las cuales el 24,97\% (596 ocurrencias) corresponde a la construcción comparativa intensificadora. Esto quiere decir que tres cuartas partes de los resultados no están licenciados por esta construcción, sino por otras: la construcción comparativa gramatical, interpretable en sentido literal (vid. 10), la construcción de relativo, con que como relativo (vid. 11), la construcción intensificadora concesiva de modalidad epistémica [por más ADJ que VERBO] (vid. 12) o la construcción [nada es más $\mathrm{X}$ que $\mathrm{Y}$ ] con el significado de ' $\mathrm{Y}$ posee la máxima cualidad posible respecto a $\mathrm{X}$ ' (vid. 13), entre otras posibilidades menos frecuentes.

(10) Y entonces además de solo te sientes tonto, o sea más tonto que antes (y quizás más solo, solamente una persona -y nadie ni nada más-, todavía un individuo...). [SkE 15841593368]

(11) La idea de que los niños quieren dormir con sus madres le parece a la mayoría de los hombres la cosa más tonta que han oído. Obviamente, no le pareció así a Freud, quien escribió que cuando era niño una vez tuvo una reacción erótica al ver a su madre vistiéndose. [SkE 744889869]

(12) Escuchar que tampoco disfrutaba de la soledad le hacía sentir algo cálido en su cuerpo, estaban solos en compañía, por más tonto que sonase. Comenzamos a caminar uno al lado del otro por las calles. [SkE 15514531432]

(13) Nada se siente más tonto que romper el teléfono de pura negligencia y estupidez. Nadie lo sabe mejor que yo. [SkE 15696700783]

De las 596 ocurrencias licenciadas por la construcción, 123 (20,64\%) son microconstrucciones, es decir, construcciones saturadas léxicamente y lexicalizadas a partir de la construcción semiesquemática [ser más tonto que X]. Se trata de los types: Abundio (66 ocurrencias), Pichote (29), hecho por encargo (15), el que asó la manteca (4), Capirote (3), Perico el de los Palotes (2), hostia (2), Carracuca (1) y yo qué sé (1). Esto significa que casi un $80 \%$ de las instancias emitidas por el hablante son creaciones ad hoc y no microconstrucciones "prefabricadas". Además, ni siquiera las 123 instancias de construcciones lexicalizadas y saturadas léxicamente están libres de variación, presentando 22 de ellas $(17,89 \%)$ algún tipo de extensión analógica.

Las construcciones creativas, por su parte, tienen una mayor presencia en el corpus con un total de 473 ocurrencias $(79,36 \%)$, es decir, no se tratan de instancias a partir de formas lexicalizadas, sino que son instancias creativas licenciadas a partir de la construcción semiesquemática [ser más tonto que X]. Teniendo en cuenta que la productividad "is measured in the context of a construction to determine how many different items occur in the various schematic slots of a construction" (Boas 2013: 247; cfr. Bybee 2013: 62), se contabilizan un total de 245 ítems léxicos diferentes que, junto con los nueve types anteriores suma un total de 254 types $(40,62 \%)$, lo que contribuye a la fijación cognitiva (entrenchment) y la convencionalización de la construcción fraseológica. Estrechamente relacionado con la frecuencia de un type, otro factor que incide en la productividad de una construcción es el número de ocurrencias hápax o con una 
frecuencia absoluta muy baja (Zeschel 2012: 174). Así pues, el corpus arroja un total de 189 hápax, como por ejemplo pepino, gallina de noche, saco de orcos, prepucio, arbusto, ameba, patata, lechuga, cigüeña, ceporro, calamar de silicona, testigos de Jehová, hombre monógamo, plátano, baldosas, libros, pájaros, Lepe.

Esta construcción fraseológica, al igual que otras, como [ser más feo que X] (vid. Ivorra Ordines en prensa) puede considerarse como especialmente productiva dado el amplio espectro de tipos de actualizaciones del slot X, en total 486 types distintos $(43,86 \%)$ de 1108 ocurrencias (vid. apartado 2.3), lo que corrobora también su fijación cognitiva y prueba su alto grado de esquematicidad. Asimismo, el elevado número de hápax (455), es de igual modo un índice de la productividad y de la creatividad que los hablantes muestran al hacer uso de esta construcción.

\subsection{Significado y función de la construcción}

De acuerdo con el DRAE, el adjetivo tonto/- $a$ se puede aplicar a personas y a cosas. Entre las acepciones que presenta, son dos las que aparecen representadas en el corpus: (i) 'dicho de una persona: falta o escaso de entendimiento o razón' (vid. 5-7) y (ii) 'que carece de sentido o de motivo' (vid. 14), siendo la primera opción la mayoritaria en una proporción del $88,94 \%$ (531 ocurrencias).

(14) Esto es más tonto que asar la mantequilla, a quién se le ocurre fichar al tipo este para hacer la publi de Nocilla, hasta que no quiten al sujeto este no la pruebo. [SkE 5509062801]

La función de esta construcción consiste, como ya se comentó más arriba, en la intensificación de la cualidad de tonto (aplicada a personas o cosas) hasta su máximo nivel, es decir, se trata de una intensificación del dictum. Asimismo, al elegir este predicado evaluativo cuantificador, el hablante emite un juicio subjetivo de valor epistémico sobre el contenido proposicional (cfr. Haverkate 1995 y 2002), esto es, tiene lugar también una intensificación de la modalidad (vid. supra apartado 1). La construcción presenta igualmente una función perlocutiva, ya que el hablante busca ejercer una influencia sobre el oyente y convencerle de lo dicho en el enunciado. Para lograr este fin, actualiza el slot libre o bien por medio de unidades ya lexicalizadas o bien improvisa él mismo una actualización del slot en el momento mismo del discurso. Las creaciones ad hoc, normalmente con tono humorístico, representan para el hablante un estereotipo de estulticia y se integran por lo general en alguno de los siguientes campos conceptuales?:

7 No hemos tenido en cuenta dentro de esta taxonomía tres instancias con aposiopesis ([más tonto que ...], [más tonto que ...] y [más tonto que un ...]), dos instancias con interjecciones ([más tonto que ojú] y [más tontos que ojú]) y dos instancias con eufemismos mediante asteriscos o puntos suspensivos ([más tontos que una ******] y [más tonto que una $M \ldots]$ ). 
Más tontos que el novio de la Chelo. La intensificación de la estulticia en foros y chats por medio de comparaciones creativas: una aproximación desde la gramática de construcciones

a) Personajes reales o inventados, considerados por el hablante como prototipos de la estulticia. Pueden ser nombres propios o no (vid. infra): 224 ocurrencias $(37,59 \%)^{9}$.

b) Animales ${ }^{10}$ que para el hablante representan prototipos de la estulticia (59 ocurrencias, 9,9\%).

c) Objetos especialmente simples y sin mucho valor, y que por ello el hablante quizás asocie analógicamente a la falta de intelecto (223 ocurrencias, 37,42\%).

d) Acciones carentes de sentido para el hablante: 63 ocurrencias (10,57\%).

\section{Actualizaciones léxicas del slot X}

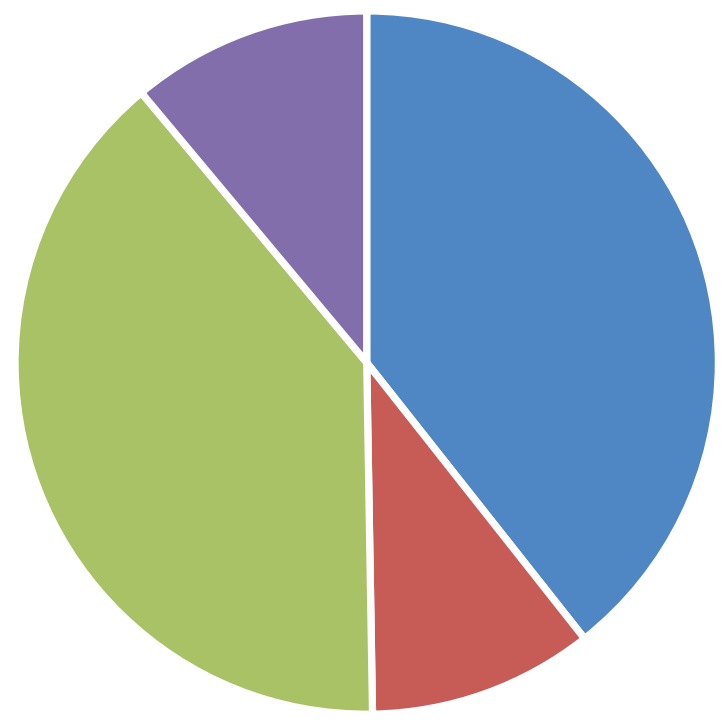

- Personajes reales o inventados - Animales

- Objetos simples

- Acciones carentes de sentido

Tabla 1. Campos conceptuales en la actualización léxica creativa del slot X.

8 Cuando no se trata de nombres propios, la actualización del slot $\mathrm{X}$ suele aludir a personas que encierran de alguna manera una contradicción desde la perspectiva del hablante, p.ej.: obrero de derechas, inmigrante racista, obrero nacionalista.

9 Habría que incluir en este campo, así como en el campo d), veinte instancias que pueden pertenecer a ambos (vid. 19-20).

$10 V a c a$, pez, orco con un garrote nuevo, perro, avestruz, monos, cigüeña, paloma, koala, sapos camuflados de príncipes, gallinas de noche, cabra, mula, hámster, burro, hormigas, oveja, ratón, mosquito lobotomizado, lagartija, cerdo facotero, cangrejo con boina, faja y anteojos, pulpo Paul, palomas, ameba, berberecho, etc. 
A continuación, vemos algunos ejemplos de cada campo conceptual:

(15) Son como los progres del fútbol. Van de listos y son más tontos que Panete y Pichote haciendo un dúo. El prestigioso diario neoyorquino defiende la figura del entrenador del Real Madrid frente a la de otros técnicos con más cartel. [SkE 4366492394]

(16) Creo que somos más tontos que un koala (al parecer es un caso raro de animal que involuciona en lugar de evolucionar ${ }^{11}$ o simplemente queremos soñar mundos imposibles, pero eso sí, que sean reales, ¿eh? [SkE 5579919746]

(17) Como dicen [...], el que se piense que esto es más "eficiente" es más tonto que un botijo, excepto si es accionista de Indra. En Canarias, la gente puede usar el teléfono para concertar una cita en la Seguridad Social, pero cobran unos céntimos por la llamada. [SkE 13727341991]

(18) Le pregunta, sí le contesta Bolín (mirando a la cámara de al lado) mientras piensa: si se lo cree, es más tonto que el gato de mi vecino que se come las margaritas y se cree que son huevos fritos. El muchacho al decirle Bolín que, si sabe clasificar pescado, se pone muy contento y le dice, iquieres ganarte un buen sueldo? [SkE 4828463074]

En no pocos casos se da una combinación de los dominios a), b) o c) con d), como en (19-20):

(19) Seguramente tenía tan poca vida ya, que poco le importaba perder un riñón a cambio de más tecnología. Si no, es más tonto que la Esteban hablando de historia. Pensaba que con el tema crisis el consumismo por consumir se había acabado por lo menos tales extremos... pero ya veo que no. [SkE 5736095402]

(20) Es preferible un mal menor a otro mayor o en el campo que es preferible el humo de la hoguera al frío de la intemperie. Es más tonto que Abundio, que fue a vendimiar y se llevó uvas de postre: Expresa el colmo de la simpleza y la bobería. [SkE 8027710180]

El efecto de las actualizaciones léxicas que designan objetos inanimados (dominio c) resulta especialmente absurdo, por carecer estos de intelecto. De acuerdo con Moreira Flores (2004) y Mellado Blanco (2012a), cuanto más absurda resulta la comparación, más expresiva y relevante es desde un punto de vista comunicativo y pragmático, y mayor es también la incompatibilidad lógico-semántica entre el tertium comparationis y el comparatum. Así se explica la presencia de secuencias como más tonto que un bocata de pipas/que un botijolque una suela de un zapatolque un puñado de piedras/que una pared de ladrillolque un saco de berzas/que una caca de pavo, etc. En algunos casos, el objeto aludido está defectuoso y no puede cumplir su función principal: más tonto que una tiza negralque un lápiz sin puntalque un vibrador sin baterías/que un boli bic sin tintalque una

11 En este ejemplo es interesante la reflexión metalingüística del hablante para justificar la elección del koala como prototipo de la estulticia. Esto muestra que el hablante es consciente del valor de estereotipo que debe tener la actualización léxica del slot $\mathrm{X}$ de la construcción para que esta cumpla su función intensificadora. 
Más tontos que el novio de la Chelo. La intensificación de la estulticia en foros y chats por medio de comparaciones creativas: una aproximación desde la gramática de construcciones

casa sin ventanas, etc. De igual manera, las acciones a las que alude la actualización léxica de X (campo conceptual d) carecen de lógica y/o son difícilmente ejecutables en la vida real: más tonto que parido por teléfonolque pellizcar cristales/que un bocado en el pielque el que vendió el coche para comprar gasolinalque cagar con capalque mear para arribalque escupir hacia dentrolque vender el tractor para comprar gasolinalque vender la burra para comprar cebadalque ir a vendimiar y llevarse uvas de postre, etc.

Especial atención merecen entre los objetos y las acciones mencionados (campos c-d) las alusiones escatológicas (vid. 21-23) o sexuales (vid. 24):

(21) Son más tontos que los pelos del culo, que ven caer la mierda y no se apartan... La verdad que los precios de mil anuncios asustan, algo más nuevo de 2005 no baja de 30000 y después tener suerte de que salga bien. [SkE 13652135802]

(22) O sea, que Poyales es más tonto que mear en un porrón. Allí no puedes decir eso de "hazle una corrección fraterna" porque si es tonto es tonto, y donde no hay mata no hay patata. [SkE 6314437686]

(23) Tú habrás ido al instituto y a la universidad, pero aún así eres más TONTO que el cagar tumbado. Te lo explico de forma clara y sencilla, a ver si así un idiota como tú puede comprenderlo. [SkE 20037271816]

(24) Es más tonta que un bocado en la polla y cada vez que habla sube el pan. Pero es una máquina entrenando. [SkE 17984062555]

Este tipo de imágenes soeces suponen un refuerzo de la expresividad y del efecto humorístico. Son índice de un registro muy coloquial o, incluso, vulgar. Algunos de estos types escatológicos son recurrentes, como los pelos del culo, con distintas variantes (en total 12 ocurrencias), a pesar de no aparecer en ninguno de los diccionarios consultados. También se registran varias ocurrencias de los verbos mear (8) y cagar (12), con distintas variaciones.

Como ya hemos indicado, en el 20,64\% de las ocurrencias extraídas del corpus se registran actualizaciones de $\mathrm{X}$ que están lexicalizadas. Se trata en concreto de los types: Abundio (66 ocurrencias), Pichote (29), hecho por encargo (15), el que asó la manteca (4), Capirote (3), Perico el de los Palotes ${ }^{12}$ (2), Carracuca (1), hostia (2) y yo qué sé (1). Entre las comparaciones lexicalizadas destacan en número los nombres propios de persona, si bien Capirote y Pichote son falsos nombres propios. El primero procede del nombre común capirote $^{13}$ (ser tonto de capirote), el segundo del italiano picciotto $^{14}$. La preponderancia de

12 De acuerdo con el DRAE, Perico de los palotes o el de los palotes se refiere a una "persona indeterminada". En el corpus aparece como variación creativa también el falso nombre propio Palote.

13 Capirote se refiere a la "capucha antigua con falda que caía sobre los hombros y a veces llegaba a la cintura" (DRAE). Según Correas, en su Vocabulario de Refranes, los bobos de capirote son llamados así "porque es ordinario ponérsele (el capirote) por burla" (vid. Iribarren 1993: 80).

14 Parece que Pichote proviene del italiano picciotto ('muchacho'), apodo de uno de los gangsters enemigos de Al Capone durante la guerra de bandas de los años 20 del S. XX: Gennaro "Il picciotto" Spummarolo, llamado así por su aspecto juvenil. Se dice que para preparar el asesinato de Richard Lonergan, Gennaro fue engañado de una forma extremadamente ingenua, de ahí que surgiera el prototipo de la estulticia más tonto que Pichote (vid. https://emitologias.wordpress.com/2013/11/12/ser-mas-tonto-que-pichote-origen/). 
los nombres propios como estereotipo de la estulticia en las comparaciones lexicalizadas actúa como trigger o desencadenante -de acuerdo con la terminología de la Gramática de Construcciones- para la creación analógica de nuevas actualizaciones del slot X. Los nombres de las comparaciones estereotipadas se refieren a personajes que con toda probabilidad son imaginarios ${ }^{15}$ (Abundio, Carracuca, Perico el de los Palotes). Las extensiones analógicas creativas aluden o bien a personajes reales (Marta Sánchez, Messi, el pulpo Paul, Dani Martínez, Florentino Fernández, Yola Berrocal, Zapatero), a personajes literarios o de ficción (la Adonis de Praxila, el burro de Sancho Panza, Espinete, Mortadelo, Snoopy, el del turrón El Almendro), o bien a personajes inventados (Afilamazas, Comín, Frasquito, Pinito, Prepucio, el hombre de la alcayata). La inclusión de este tipo de nombres responde en todos los casos a una intención humorística y expresiva ${ }^{16}$.

Los ejemplos citados corroboran la preponderancia cognitiva de los nombres propios e individualizadores dentro de esta construcción (campo conceptual a), vid. supra), que en el corpus se materializa expresamente en una de las actualizaciones del slot X:

(25) Sí, estamos lidiando con gente más tonta que el personaje ficticio más tonto. Las autoridades de Arizona han pedido ayuda para localizar a los caballeros en esa foto después que alguien vandalizó un sitio ritual indio norteamericano de 800 años en Sedona, Arizona, al arrojar sus contenidos en un dique cercano. [SkE 8179783113]

Por último, y como prueba del efecto de coerción que ejerce toda la construcción sobre los componentes parciales de la misma, algunas actualizaciones léxicas del slot $\mathrm{X}$ pueden considerarse como comodines, es decir, son intercambiables con otras microconstrucciones intensificadoras. Se trata de los llamados comparata "passepartout" (cfr. Luque Durán 2005: 425-426; Mellado Blanco 2012a: 25-26), del tipo Carracuca, la hostia (vid. ejemplo 3), yo qué sé, lexicalizados y combinables con numerosos tertia comparationis.

\subsection{Creatividad de la construcción: relaciones horizontales}

Uno de los objetivos principales de la lingüística moderna es poder explicar el potencial creativo del lenguaje, teniendo en cuenta que permitir que las construcciones

15 Buitrago (2007), en relación con el nombre de Carracuca, apunta que es uno de esos personajes proverbiales de "filiación desconocida" que se usa como paradigma de lo negativo (cita también en este sentido más feo que el sargento de Utrera y más tonto que Abundio).

16 La tendencia a incluir nombres propios, reales o ficticios, no es exclusiva de esta construcción comparativa. También aparece en otras, por ejemplo, las de polaridad negativa basadas en una antítesis, del tipo Ese tiene de mago lo que yo de Bill Gates: 'Ese no es mago de ninguna manera' (vid. Mellado Blanco 2015 y 2019). En ciertas ocasiones, las construcciones comparativas con nombres propios pueden ejercer la función indirecta de criticar a un determinado personaje real a través de la cualidad negativa atribuida en grado sumo, como se comprueba en nuestro corpus en relación con Belén Esteban (vid. ejemplo 19) y George McFly (ejemplo 4). Observamos, a su vez, nombres propios en otras comparaciones basadas en la ironía, como más feo que Rita Hayworth: 'muy guapo', como recurso elativizador en cuanto acto afirmativo que niega (vid. Ivorra Ordines en prensa). 
Más tontos que el novio de la Chelo. La intensificación de la estulticia en foros y chats por medio de comparaciones creativas: una aproximación desde la gramática de construcciones

se puedan combinar libremente permite, a su vez, explicar dicha creatividad. Desde la Gramática de Construcciones, la noción de 'creatividad' se asocia con la productividad de una construcción, es decir, "how established abstract schemas of a language can be used to license novel utterances" (Hoffmann 2019; cfr. Goldberg 2019).

Como veíamos en el apartado anterior, la creatividad puede darse a partir de construcciones lexicalizadas o microconstrucciones. De esta manera, una alta frecuencia token puede ser un indicador de productividad o creatividad para las construcciones menos esquemáticas, mediante las cuales se licencian extensiones analógicas, puesto que "highly entrenched tokens are more easily activated than non-entrenched tokens, and are thus more available as model items than infrequently occurring tokens" (Barđdal 2008: 98). A partir de la microconstrucción [ser más tonto que Abundio] con una alta frecuencia token (66 ocurrencias en el corpus), se licencian un total de 14 extensiones analógicas mediante la expansión del comparatum para enfatizar el significado fraseológico de la construcción y, de este modo, contrarrestar el desgaste expresivo de comparaciones fraseológicas que se usan con frecuencia. Desde un punto de vista cognitivo, las extensiones analógicas hacen referencia a acciones que carecen de lógica o van en contra del sentido común (vid. 26-27).

(26) Como decía un chiste... No hay prisión que admita tanta fealdad. La primera pregunta que me asalta: este tío es más tonto que Abundio, que mira que Abundio era tonto de cojones. No disfrace, señora Artal, a la de Pamplona de supervíctima, porque no lo es. [SkE 17888692348]

(27) Era tan tonto el pobre Abundio que siempre que tú hagas algo absurdo te pueden comparar con él y decirte: "Eres más tonto que Abundio, que para poder comprar los cordones vendió los zapatos". Y de esto trata el octavo de Los Juegos De MiAC, de que pongáis en este tema las frases que conozcáis sobre lo tonto que era Abundio o que las inventéis vosotros mismos. [SkE 19741181182]

Otro tipo de creatividad es el que tiene lugar en la contaminación de dos o más unidades fraseológicas. Desde la Fraseología tradicional se las considera como lapsus o modificaciones no intencionadas - un tipo de desautomatización no intencionada según Martí Sánchez (2015)-, mientras que, en el marco de la Gramática de Construcciones, las contaminaciones son una consecuencia lógica de las interrelaciones que mantienen entre sí las construcciones. Hablamos pues de relaciones horizontales entre construcciones, esto es, además de las relaciones verticales en forma jerárquica, las construcciones también se pueden relacionar en el eje horizontal, de manera que "a particular construction may be partly motivated in relation to its neighbours" (Van de Velde 2014: 147; cfr. Diessel 2019: 63-66). Las contaminaciones pueden tener lugar con otras comparaciones estereotipadas (vid. 28-32) o, en general, con otras locuciones (vid. 33-34).

(28) Luego la historieta de la madre que cae en coma, [...] tiene como único objetivo hacer desgraciada a la pobre Lorena, que bastante tiene con estar enamorada de 
Alonso, que es más tonto que un dolor ${ }^{17}$, y que se la hace un día sí y otro también, pero con el que Lorena se acabará casando al final porque para eso es el galán, aunque cualquier chica normal preferiría a Ernesto. [SkE 20072216651]

(29) Que ya te conocemos, insultas a todos. ( $\mathrm{Si}$ así te crees mejor, es que eres más tonto que Lepe ${ }^{18}$ ). Además, dime de qué te jactas y te diré de qué careces. Y tú, desde el momento que no respetas debatiendo sin insultar, pierdes todo posible argumento. [SkE 376934867]

(30) Felicidades, Ana_MS y la otra Ana que viene a veces. Y ya me paro que versificar tanto me saldrá caro: dirán que es vicio, y que parezco más tonta que Picio ${ }^{19}$. ¡Vamos, vanidosa yo! Si tenía que rimar con "hermosa" ¿no tenía "preciosa”, por ejemplo? [SkE 3562969821]

(31) Estáis esclavizados [...] en la segunda parte del siglo XIX, al parecer sin demasiados escrúpulos de conciencia. Ya empezamos a estar hartos. Y los voceros oficiales (más tontos que Calleja ${ }^{20}$ ) vendiéndonos la burra ciega de que la Eta está derrotada (ja, ja...). El asesinato para la Eta era un medio. [SkE 13540095953]

(32) Como buen flash movie que se precie, cae en dos tópicos y un fallo muy propio del género. Tópico 1: Para que el villano sea competente, los polis deben ser más tontos que un $\operatorname{arado}^{21}$. Tópico 2: Como no podía ser de otra forma, una vez que se deja inconsciente al villano, no se le remata. [SkE 20224209476]

(33) He investigado en Facebook y no me extrañaría que aparezcan Fan Pages hablando del tema. No he querido hacerme fan de "Más alta que un pino, más tonta que un pepino $^{22}$ " por temor a represalias. Hemos sido culpados de provocar intoxicaciones sin razón aparente alguna, por lo que parece, y Twitter reacciona. [SkE 7237522743]

(34) Mira que sois más tontos que los tontos de pueblo ${ }^{23}$. Estamos entrando de lleno en lo que va a ser el cambio climático, que ya está asegurado y se os ocurre hablar de idiomas en estos momentos, de desastre planetario. Idiotas, os hundiréis todos como el Titanic. [SkE 6376627691]

17 Como un dolor (o más que un dolor): "Constr de sent comparativo usada para ponderar la fealdad o la maldad" (Seco, Andrés y Ramos 2017: 286).

18 Saber más que Lepe: "Tener gran perspicacia y astucia. A veces incrementado: Lepe, Lepijo y su hijo. Con intención ponderativa" (Seco, Andrés y Ramos 2017: 452).

19 Más feo que Picio: "Sumamente feo. Gralm referido a pers." (Seco, Andrés y Ramos 2017: 653). Vemos en este caso un ejemplo de coerción fonética: teniendo en cuenta la intencionalidad del hablante de rimar 'vicio' con el ítem léxico que actualiza el slot $\mathrm{X}$, se produce la contaminación con otra comparación estereotipada con claros fines poéticos.

20 Tener más cuento que Calleja: "Tener imaginación o fantasía para urdir o inventar. Gralm con intención enfática" (Seco, Andrés y Ramos 2017: 232).

21 Más bruto que un aradolmás burro que un arado: "Constr de sent comparativo usada para ponderar lo bruta que es una pers." (Seco, Andrés y Ramos 2017: 45).

22 Un pepino: "Nada. Tb adv. Con intención ponderativa. Gralm con vs como valer o importar" (Seco, Andrés y Ramos 2017: 642). Nos encontramos aquí con el mismo caso de coerción fonética que en el ejemplo 30 , aunque en este caso la rima se produce entre 'pino' y 'pepino'. Para un estudio más exhaustivo de los términos de polaridad negativa del tipo un pepino vid. Mellado Blanco (2020b).

23 "El tonto del pueblo o bobo del pueblo es, en sentido estricto, una persona conocida localmente por su ignorancia o estupidez, pero es también un término común para una persona estereotípicamente tonta o sin sentido común” (https://es.wikipedia.org/wiki/Tonto_del_pueblo). 
Más tontos que el novio de la Chelo. La intensificación de la estulticia en foros y chats por medio de comparaciones creativas: una aproximación desde la gramática de construcciones

Estas contaminaciones constituyen una interesante prueba de la flexibilidad cognitiva tanto del hablante, que no se ciñe a unidades lexicalizadas para actualizar la construcción, como del oyente, al que no le cuesta interpretar y reconocer estas actualizaciones híbridas como instancias de la construcción intensificadora. De ello podemos inferir que el hablante, en el acto de comunicación, tiende a transferir, a modo de comodín, secuencias lexicalizadas de determinados tipos de construcción a instancias licenciadas por construcciones distintas (cfr. Mellado Blanco 2020a en relación con los "lapsus"). Además, las contaminaciones evidencian la frontera difusa que existe entre el error y la modificación creativa, distinción que no resulta esencial desde un enfoque construccionista. El fenómeno de la contaminación responde más bien a asociaciones cognitivas entre diferentes construcciones; en otras palabras, "a tangled ball of wool, where individual threads cannot be isolated easily, as they are inextricably entangled in other threads, and several may even get interwoven into a single thread" (Pijpops y Van de Velde 2016: 549).

\section{A modo de conclusión}

El análisis basado en corpus nos ha permitido constatar la existencia de dos dominios léxicos que sirven de trigger o catalizador en las formaciones creativas por medio de extensiones analógicas. Tanto nombres propios (Abundio y Pichote), como acciones absurdas o carentes de lógica (hecho por encargo, el que asó la manteca) sirven de trigger en la actualización del slot $\mathrm{X}$ y, por ende, en la creatividad de la construcción, tal y como se pone de manifiesto en la clasificación de los campos conceptuales que se utilizan como estereotipos de la estulticia. Estas analogías subrayan que las nuevas ocurrencias (creativas) están basadas en ejemplares existentes, hecho que indica que la memoria es significativamente asociativa en la medida que "new knowledge is integrated with prior knowledge $[\ldots]$ and each memory trace is therefore inextricably linked to other, related memory traces" (Goldberg 2019: 52; cfr. Bybee 2010 y 2013).

En esta misma línea de razonamiento, observamos que si aplicamos el principio escalar de Fauconnier (1975) a la construcción fraseológica [ser más tonto que X]: 'ser muy tonto', de la misma manera que se puede aplicar al resto de construcciones comparativas intensificadoras, los ítems léxicos que actualizan el slot $\mathrm{X}$ representan elementos que cultural o subjetivamente, desde el punto de vista del hablante, se sitúan en el punto más alto de la escala de la estulticia y, por inferencia, todo lo que alude ese comparatum $\mathrm{X}$ es valorado como lo más tonto posible, es decir, como la cualidad de tonto en grado sumo. Este hecho permite explicar, a pesar de la alternancia entre lo lexicalizado y lo creativo, el efecto semántico que se produce al mantenerse la interpretación independientemente del ítem léxico elegido.

En el contexto de la Gramática de Construcciones basada en el uso, la creatividad se concibe como un fenómeno intrínseco a la lengua, de tal manera que las formas creativas son instancias menos prototípicas que las consideradas como lexicalizadas, llegando algunas de ellas a aparecer tan solo una vez en el corpus (hápax), las cuales pueden resultar más "exóticas" en algunos casos atendiendo a las grandes dimensiones 
del corpus que hemos utilizado (vid. ejemplos 18, 20 y 21). Uno de los procedimientos creativos que hemos constatado se basa en la elaboración de slots a partir de una construcción totalmente saturada. De este modo, dos o más instancias de un slot dentro de una construcción conducen a la formulación de una microconstrucción que tiene la capacidad de licenciar extensiones analógicas. Dentro del contínuum léxico-gramática, dichas microconstrucciones se sitúan más cerca del polo léxico por considerarse más "fraseológicas". En este contexto, una alta frecuencia token se concibe como un factor esencial cuando hablamos de creatividad en las construcciones sustantivas, como sería el caso de la microconstrucción [ser más tonto que Abundio]: 'ser muy tonto', la cual, con un total de 66 ocurrencias en el corpus, licencia 14 extensiones analógicas con una clara finalidad expresiva a través de acciones carentes de lógica. Este tipo de creatividad, desde un enfoque construccionista, responde a relaciones verticales de herencia, de manera que en una red taxonómica las microconstrucciones con extensiones analógicas heredarían las propiedades de las microconstrucciones saturadas léxicamente y, a su vez, ambas heredarían las propiedades de la construcción semiesquemática [más tonto que X]: 'muy tonto'. En esta misma línea, a partir de la definición de la gramática como "network of constructions [that] captures our grammatical knowledge of language in toto" (Goldberg 2006: 18), las relaciones horizontales entre las construcciones permiten explicar la tendencia a tener miembros de diferentes fuentes y, de este modo, ofrecen una visión más detallada de las relaciones que existen entre las construcciones que la que pueden dar solo los modelos verticales (Traugott 2018: 20). Por lo tanto, dichas relaciones ilustran la contaminación entre diferentes unidades fraseológicas que comparten una semántica o pragmática similar (locución y comparación o comparación y comparación), poniendo de manifiesto los cambios constantes a los que está sometida la lengua en general, y las unidades fraseológicas en particular.

En resumen, la Gramática de Construcciones se presenta como un marco teórico idóneo y flexible para el estudio del binomio 'intensificación'/'comparación', constatándose así el predominio de lo creativo sobre lo lexicalizado (79,36\% frente al $20,64 \%$, respectivamente) y el amplio abanico de posibilidades creativas que dispone el hablante a la hora de expresar la estulticia en grado máximo.

\section{BIBLIOGRAFÍA}

ALBELDA MARCO, Marta (2007): La intensificación como categoría verbal plena: revisión y propuesta. Una aplicación al español coloquial. Frankfurt: Peter Lang.

BARĐDAL, Jóhanna (2008): Productivity. Evidence from Case and Argument Structure in Icelandic. Amsterdam: John Benjamins.

BEINHAUER, Werner (1985): El español coloquial. Madrid: Gredos.

BEISSWENGER, Michael (2003): „Sprachhandlungskoordination im Chat“, Zeitschrift für germanistische Linguistik. Vol. 31/2: 198-231.

BOAS, Hans (2013): "Cognitive Construction Grammar", Thomas Hoffmann; Graeme Trousdale (eds.), The Oxford Handbook of Construction Grammar. Oxford: Oxford University Press, 233-252. 
Más tontos que el novio de la Chelo. La intensificación de la estulticia en foros y chats por medio de comparaciones creativas: una aproximación desde la gramática de construcciones

BUITRAGO, Alberto (2007): Diccionario de dichos y frases hechas. 5000 dichos y frases hechas diferentes y 3000 variantes de los mismos. Madrid: Espasa.

BYBEE, Joan (2010): Language, Usage and Cognition. Cambridge: Cambridge University Press.

(2013): "Usage-based Theory and Exemplar Representations of Constructions", Thomas Hoffmann; Graeme Trousdale (eds.), The Oxford Handbook of Construction Grammar. Oxford: Oxford University Press, 49-69.

CIFUENTES HONRUBIA, José Luis (2019): "Construcciones con minimizadores y verbos de estima o valoración y el ciclo de Jespersen", Revista de Investigación Lingüística. Vol. 22: 53-94.

DIESSEL, Holger (2019): "Usage-based construction grammar", Ewa Dabrowska; Dagmar Divjak (eds.), Cognitive Linguistics - A Survey of Linguistic Subfields. Berlín: De Gruyter, 50-80.

FAUCONNIER, Gilles (1975): "Pragmatic Scales and Logical Structure", Linguistic Inquiry. Vol. 6/3: 353-375.

FILLMORE, Charles; KAY, Paul; O'CONNOR, Mary Catherine (1988): "Regularity and Idiomaticity in Grammatical Constructions: The Case of Let Alone", Language. Vol. 64/3: 501-538.

GOLDBERG, Adele (1995): A Construction Grammar Approach to Argument Structure. Chicago: The University of Chicago Press.

(2006): Constructions at Work. The Nature of Generalization in Language. Oxford: Oxford University Press.

(2019): Explain me this. Creativity, Competition and the Partial Productivity of Constructions. Princeton University Press.

HAVERKATE, Henk (1995): "Spanish mood and the expression of cognitive and evaluative meaning", Verba. Vol. 22: 11-29.

(2002): The Syntax, Semantics and Pragmatics of Spanish Mood. Amsterdam: John Benjamins.

HOFFMANN, Thomas (2019): "Language and Creativity. A Construction Grammar approach to linguistic creativity", Linguistic Vanguard. Vol. 5/1.

IRIBARREN, José (1993): El porqué de los dichos: Sentido, origen y anécdota de dichos, modismos y frases proverbiales. Pamplona: Ariel.

IVORRA ORDINES, Pedro (en prensa): "Comparative constructional idioms. A corpus-based study of the creativity of the [más feo que X] construction", Carmen Mellado Blanco (ed.), Productive Patterns in Phraseology and Construction Grammar. A Multilingual Approach. Berlín: De Gruyter, 29-52.

(2021): "De comparaciones fraseológicas a construcciones fraseológicas: el rol de las microconstrucciones emergentes en la creación de comparaciones fraseológicas", eHumanista IVITRA. Vol. 19: 440-455 [https://www.ehumanista.ucsb. edu/sites/default/files/sitefiles/ivitra/volume19/06.\%20Ivorra.pdf; 30/06/2021]

KLEINBERGER GÜNTHER, Ulla (2006): „Phraseologie und Sprichwörter in der digitalen Öffentlichkeit - am Beispiel von Chats", Annelies Häcki Buhofer; 
Harald Burger (coords.), Phraseology in Motion I. Methoden und Kritik. Baltmannsweiler: Schneider Verlag Hohengehren, 229-243.

KOCH, Peter; ÖSTERREICHER, Wulf (1985): „Sprache der Nähe - Sprache der Distanz. Mündlichkeit und Schriftlichkeit im Spannungsfeld von Sprachtheorie und Sprachgeschichte", Romanistisches Jahrbuch. Vol. 36/85: 15-43.

LAROUSSE (2001): Gran diccionario de frases hechas. Barcelona: SPES Editorial.

LUQUE DURÁN, Juan de Dios (2005): "Las colocaciones de cuantificación por comparación: tradición e innovación en las comparaciones proverbiales", Juan de Dios Luque Durán; Antonio Pamies (eds.), La creatividad en el lenguaje: colocaciones idiomáticas y fraseología. Granada: Granada Lingvística, 409-456.

MARTÍ SÁNCHEZ, Manuel (2015): "La búsqueda de sentido en la desautomatización fraseológica", Pedro Mogorrón; Fernando Navarro (eds.), Fraseología, Didáctica y Traducción. Frankfurt am Main: Peter Lang, 117-135.

MELLADO BLANCO, Carmen (2012a): "Las comparaciones fijas en alemán y español: algunos apuntes contrastivos en torno a la imagen", Linred: Lingüistica en Red. Vol. 10: 1-32. [http://www.linred.es/monograficos_pdf/LR_monografico10-articulo1.pdf; 22/12/2020]

(2012b): "Optimización de los recursos TIC en la fraseografía del par de lenguas alemán-español”, María Isabel González Rey (ed.), Unidades fraseológicas y TIC. Madrid: Instituto Cervantes (Biblioteca fraseológica y paremiológica, $\mathrm{n}^{\circ}$ 2), 147-166. [https://cvc.cervantes.es/lengua/biblioteca_fraseologica/ n2_gonzalez/mellado.htm; 22/12/2020]

(2015): "Antiphrasis-based Comparative Constructional Idioms in Spanish", Journal of Social Sciences (Special Issue Phraseology, Phraseodidactics and Construction Grammar(s). Vol. 11/3: 111-127. [http://thescipub.com/PDF/ jssp.2015.111.127.pdf; 22/12/2020]

(2019): "Phrasem-Konstruktionen kontrastiv Deutsch-Spanisch: ein korpusbasiertes Beschreibungsmodell anhand ironischer Vergleiche", YOP, Yearbook of Phraseology. Vol. 10: 65-88.

(2020a): "La desautomatización desde el prisma de la Gramática de Construcciones: un nuevo paradigma de la variabilidad fraseológica", Nasledje. Vol. 45: 17-34. [https://drive.google.com/file/d/1I54zjtbbGWGV0RQ425hD1FzurkYS3vaR/view; 22/12/2020]

(2020b): “(No) me importa un comino y sus variantes diatópicas. Estudio de corpus desde la Gramática de Construcciones”, ELUA. Anexo VII, 87-109. [https://rua.ua.es/dspace/bitstream/10045/109685/1/ELUA-Anexo-VII_06. pdf; 22/12/2020]

MICHAELIS, Laura (2003): "Headless constructions and coercion by construction", Elaine Francis; Laura Michaelis (eds.), Mismatch: form-function incongruity and the architecture of grammar. Standford: CSLI Publications, 259-310.

MOLINER, María (2007): Diccionario de uso del español actual. Madrid: Gredos. 
Más tontos que el novio de la Chelo. La intensificación de la estulticia en foros y chats por medio de comparaciones creativas: una aproximación desde la gramática de construcciones

MOREIRA FLORES, Cristina (2004): Zum Ausdruck des höchsten grades: im deutschen und im portugiesischen. [https://repositorium.sdum.uminho.pt/handle/1822/646; 22/12/2020]

PENADÉS MARTÍNEZ, Inmaculada (2012): Gramática y semántica de las locuciones. Alcalá de Henares: Universidad de Alcalá, Servicio de Publicaciones.

PIJPOPS, Dirk; VAN DE VELDE, Dirk (2016): "Constructional contamination: How does it work and how do we measure it?", Folia Linguistica. Vol. 50/2: 543581.

RODRÍGUEZ, Carlos J. (2000): "Entrevista a Manuel Seco Reymundo. El diccionario que no se renueva está condenado al olvido". Espéculo. Revista de estudios literarios. [http://www.ucm.es/info/especulo/numero14/m_seco.html; 22/12/2020]

SECO, Manuel; ANDRÉS, Olimpia; RAMOS, Gabino (2017): Diccionario fraseológico documentado del español actual: locuciones y modismos españoles. $2^{a}$ Edición corregida y aumentada. Madrid: JdeJ Editores.

SKETCH ENGINE: Spanish Web corpus 2018 (esTenTen18) EU + AM, Sketch Engine: Corpus Query System. [http://sketchengine.com.uk; 22/12/2020]

STEIN, Stephan (2010): „Mündlichkeit und Schriftlichkeit, phraseologisch gesehen“, Jarmo Korhonen; Wolfgang Mieder; Elisabeth Piirainen; Rosa Pinel (eds.), Phraseologie global - areal - regional. Tübingen: Narr, 409-416.

TRAUGOTT, Elizabeth Closs (2018): "Modeling Language Change with Constructional Networks", Salvador Pons Bordería; Óscar Loureda Lamas (eds.), Beyond Grammaticalization and Discourse Markers. New Issues in the Study of Language Change. Boston: Brill, 17-50.

VAN DE VELDE, Freek (2014): "Degeneracy: The maintenance of constructional networks", Roony Boogaart; Timothy Colleman; Gijsbert Rutten (eds.), Extending the Scope of Construction Grammar. Berlín: De Gruyter, 141-180.

VARELA, Fernando; KUBARTH, Hugo (1994): Diccionario fraseológico del español moderno. Madrid: Gredos.

ZESCHEL, Arne (2012): Incipient Productivity. A Construction-Based Approach to Linguistic Creativity. Berlín: De Gruyter.

\section{PERFIL ACADÉMICO Y PROFESIONAL}

Pedro Ivorra Ordines doctor en Traducción y Ciencias del Lenguaje por la Universidad Pompeu Fabra (Barcelona). Su Tesis Doctoral adopta un enfoque construccionista en el estudio de las comparaciones intensificadoras de la fealdad en diferentes lenguas. Además, es miembro del grupo de investigación FRASESPAL, en el que se está llevando a cabo el proyecto de investigación Gramática de Construcciones y Fraseología. Las construcciones fraseológicas del alemán y el español en contraste a través de corpus.

Carmen Mellado Blanco es catedrática de Filología Alemana en la Universidad de Santiago de Compostela. Está al frente desde 2007 del grupo de investigación FRASESPAL ("Fraseología del Español y el Alemán") y en este contexto ha dirigido varios 
proyectos nacionales de investigación sobre Fraseología Contrastiva y Gramática de Construcciones, el último de ellos concedido en 2019. Desde 2021 dirige junto a Fabio Mollica la revista Yearbook of Phraseology (de Gruyter), revista auspiciada por la Sociedad Europea de Fraseología (EUROPHRAS). Entre sus publicaciones más importantes como autora destacan los volúmenes Fraseologismos somáticos del alemán (Peter Lang, 2004) y el diccionario bilingüe de 35.000 unidades Idiomatik DeutschSpanisch (Buske Verlag, 2013). Como editora tiene en su haber la publicación de 12 volúmenes en editoriales internacionales de reconocido prestigio y ha suscrito más de 150 artículos y capítulos de libro relacionados con la Fraseología, la Lingüística Contrastiva, la Gramática de Construcciones, la historia de la lengua alemana y las variedades diatópicas del alemán.

Fecha de recepción: 01-03-2021

Fecha de aceptación: 20-04-21 